九州大学学術情報リポジトリ

Kyushu University Institutional Repository

\title{
Dynamic structures of the time correlation functions of chaotic nonequilibrium fluctuations
}

Mori, Hazime

Research Institute for Applied Mechanics, Kyushu University

Okamura, Makoto

Research Institute for Applied Mechanics, Kyushu University

http://hdl. handle. net/2324/1910487

出版情報: Physical Review E. 76 (6), pp.061104-1-061104-9, 2007-12-05. American Physical Society

バージョン :

権利関係 : ๑2007 American Physical Society 


\title{
Dynamic structures of the time correlation functions of chaotic nonequilibrium fluctuations
}

\author{
Hazime Mori and Makoto Okamura* \\ Research Institute for Applied Mechanics, Kyushu University, Kasuga 816-8580, Japan
}

(Received 29 May 2007; published 5 December 2007)

\begin{abstract}
Using the projection operator formalism we explore the decay form of the time correlation function $U_{n}(t)$ $\equiv\left\langle\hat{u}_{n}(t) \hat{u}_{n}^{*}(0)\right\rangle$ of the state variable $\hat{u}_{n}(t)$ in the chaotic Kuramoto-Sivashinsky equation. The decay form turns out to be the algebraic decay $1 /\left[1+\left(\gamma_{n a} t\right)^{2}\right]$ in the initial regime $t<1 / \gamma_{n e}$ and the exponential decay $\exp \left(-\gamma_{n e} t\right)$ in the final regime $t>1 / \gamma_{n e}$. The memory function $\Gamma_{n}(t)$ that represents the chaos-induced transport is found to obey the Gaussian decay $\exp \left[-\left(\beta_{n g} t\right)^{2}\right]$ in the case of large wave numbers, but the $3 / 2$ power decay $\exp \left[-\left(\beta_{n 3} t\right)^{3 / 2}\right]$ in the case of small wave numbers. The power spectrum of $\hat{u}_{n}(t)$ is given by the real part $U_{n}^{\prime}(\omega)$ of the Fourier-Laplace transform of $U_{n}(t)$ and has a dominant peak at $\omega=0$. This peak within the linewidth $\bar{\gamma}_{n e}\left(\approx \gamma_{n e}\right)$ is given by the Lorentzian spectrum $\bar{\gamma}_{n e}^{2} /\left(\omega^{2}+\bar{\gamma}_{n e}^{2}\right)$. However, the wings of the peak outside the width $\bar{\gamma}_{n e}$ turn out to take the exponential spectrum $\exp \left(-\omega / \gamma_{n a}\right)$. Thus it is found that the exponential decay $\exp \left(-\gamma_{n e} t\right)$ appears to lead to the universal Lorentzian peak, while the algebraic decay $1 /\left[1+\left(\gamma_{n a} t\right)^{2}\right]$ arises to bring about the exponential wing.
\end{abstract}

DOI: 10.1103/PhysRevE.76.061104 PACS number(s): 05.40.-a, 05.45.-a, 05.10.Gg, 02.50.Ey

\section{INTRODUCTION}

In order to clarify the transport processes generated by chaos and turbulence, such as the chaos-induced friction, we explore the decay form and the decay rate of the time correlation function of the chaotic and turbulent fluctuations that represents the average regression of the fluctuations [1-3]. Phenomenological theories of turbulent transport have been developed on the basis of both the concept of the eddy viscosity for the Reynolds stress by Boussinesq and the mixinglength theory by Prandtl, and have been used in various important fields of engineering [4]. These concepts and methods are, however, modifications of those of the kinetic theory of gases.

Therefore, if we employ the modern concepts and methods of the recent statistical physics of transport processes, such as the fluctuation-dissipation theorems and the projection operator formalism [5-8], then it would be possible to construct a more systematic and reliable theory of chaotic and turbulent transport. Indeed such a modern approach has various merits. First we deal with the time correlation functions of the hydrodynamic fluctuations by generalizing the dynamic structure factor $S(\omega, k)$ of the thermal hydrodynamic fluctuations that can be measured by the scattering of light and neutrons by the hydrodynamic fluctuations $[9,10]$. Second the projection operator formalism leads to a nonMarkovian linear evolution equation for the time correlation function that is integrable. Then the linear evolution equation is integrated to give an important relation that describes the dynamic structures of the time correlation function $U_{n}(t)$ in terms of the memory spectrum $\Gamma_{n}(i \omega)$ explicitly.

Therefore, the purpose of the present paper is to clarify the dynamic structures of the time correlation functions and the power spectra of the chaotic and turbulent fluctuations from a modern statistical-physical point of view. As a simple dynamical system, we treat the chaotic KuramotoSivashinsky (KS) equation $[1,11]$.

\footnotetext{
*okamura@ riam.kyushu-u.ac.jp
}

The present paper is organized as follows. In Sec. II, we show that the decay forms of the time correlation function $U_{n}(t)$ consist of the exponential decay and an algebraic decay. In Sec. III, the real part $U_{n}^{\prime}(\omega)$ of the Fourier-Laplace transform of $U_{n}(t)$ and the power spectrum $I_{\hat{u}_{n}}(\omega) \propto U_{n}^{\prime}(\omega)$ are written in terms of the memory spectra $\Gamma_{n}^{\prime}(\omega)$ and $\Gamma_{n}^{\prime \prime}(\omega)$. Then, in Sec. IV, the power spectrum is shown to consist of a dominant peak lying at $\omega=0$, and become Lorentzian if and only if the memory spectrum $\Gamma_{n}^{\prime}(\omega)$ has an extremum at $\omega$ $=0$. In Sec. V, we clarify the dynamic structures of $U_{n}(t)$ and $\Gamma_{n}(t)$. Then, in Sec. VI, we derive the two decay forms of $U_{n}(t)$ theoretically for the stochastic frequency modulation model. Section VII is devoted to a summary.

\section{DECAY FORMS OF THE NORMALIZED TIME CORRELATION FUNCTION $\widetilde{U}_{n}(t)$}

We treat the KS equation for the state variable $u(x, t)$ $[1,11]$

$$
u_{t}+u u_{x}+u_{x x}+u_{x x x x}=0
$$

under the boundary condition $u(x, t)=u(x+L, t)$. The spatial period $L$ is chosen to be 500, which is sufficiently large for the KS equation to produce chaotic solutions. The $N$-truncated Fourier transform of Eq. (2.1) yields $N$ evolution equations [1]

$$
\frac{d \hat{u}_{n}(t)}{d t}=L_{n} \hat{u}_{n}(t)+N_{n}(t), \quad n=1, \ldots, N
$$

where we have defined $L_{n} \equiv k_{n}^{2}-k_{n}^{4},\left(k_{n} \equiv 2 \pi n / L\right)$,

$$
\hat{u}_{n}(t) \equiv \int_{0}^{L} u(x, t) e^{-i k_{n} x} d x
$$




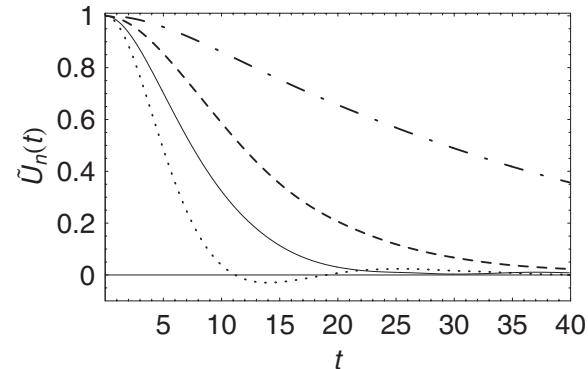

FIG. 1. The decay form of $\tilde{U}_{n}(t)$ given by the DNS of Eq. (2.6) for four modes:,$- n=55 ; \cdots, n=30 ;-\cdots, n=10 ;-\cdot-, n=5$.

$$
N_{n}(t) \equiv-\frac{i}{L} \sum_{m=-N}^{N} k_{m} \hat{u}_{n-m}(t) \hat{u}_{m}(t) .
$$

The state variable $\hat{u}_{n}(t)$ fluctuates with time according to the chaotic evolution Eqs. (2.2), leading to a statistically steady state [1]. This fluctuation $\hat{u}_{n}(t)$ is perhaps the simplest example of the turbulent hydrodynamic fluctuation.

Now let us consider the time correlation function of the fluctuation $\hat{u}_{n}(t)$ with $\left\langle\hat{u}_{n}(t)\right\rangle=0$ :

$$
\begin{aligned}
U_{n}(t) & \equiv\left\langle\hat{u}_{n}(t) \hat{u}_{n}^{*}(0)\right\rangle, \\
& =\frac{1}{M} \sum_{j=0}^{M-1} \hat{u}_{n}\left(t+T_{0}+40 j\right) \hat{u}_{n}^{*}\left(T_{0}+40 j\right),
\end{aligned}
$$

where the starting time is $T_{0}=1000$; the final time $T=10^{7}$; and the "ensemble" number $M=\left(T-T_{0}\right) / 40 \approx 2.5 \times 10^{4}$. We have used a pseudospectral method with $N=256$ for the spatial derivative and the fourth-order Runge-Kutta method with a time increment of 0.1 for the time evolution. The direct numerical simulation (DNS) of Eq. (2.6) gives the decay form of the normalized time correlation function defined as

$$
\tilde{U}_{n}(t) \equiv \frac{U_{n}(t)}{U_{n}(0)},
$$

which is shown in Fig. 1 for $n=55,30,10,5$. The angular brackets $\langle\cdots\rangle$ in Eq. (2.5) denote the long-time average (2.6). Hence Fig. 1 indicates that the mixing occurs so that $U_{n}(t)$ $=\left\langle\hat{u}_{n}(t)\right\rangle\left\langle\hat{u}_{n}^{*}(0)\right\rangle=0$ for $t \rightarrow \infty$. We find from Fig. 1 for $n=55$, 10,5 that the decay is slower as $n$ is smaller.

Figure 2 shows $\ln \widetilde{U}_{n}(t)$ against $t$ for $n=10$, indicating that

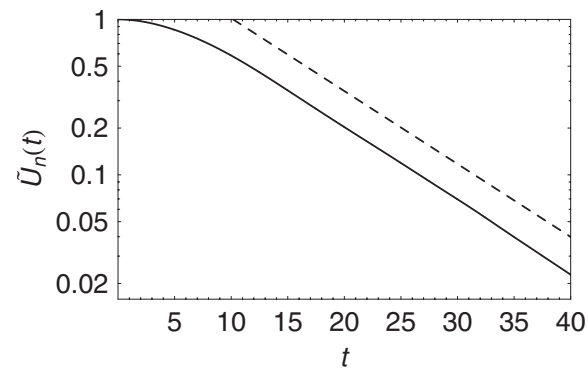

FIG. 2. $\tilde{U}_{n}(t)$ vs $t$ for $n=10$ : - - -, the exponential decay $3 \exp \left(-\gamma_{n e} t\right) ;-$, DNS, where $1 / \gamma_{n e} \approx 9.26$.

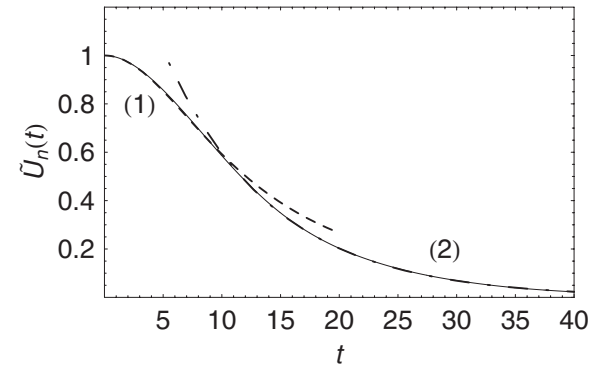

FIG. 3. A dual structure of $\widetilde{U}_{n}(t)$ for $n=10$ : (1) - - -, the algebraic decay (2.9); (2) - - -, the exponential decay (2.8); - , DNS, where $1 / \gamma_{n e} \approx 9.26$.

the tail of $\tilde{U}_{n}(t)$ is given by the exponential decay

$$
\tilde{U}_{n}(t) \simeq \alpha_{n e} \exp \left(-\gamma_{n e} t\right) \text { for } t>1 / \gamma_{n e},
$$

where $\gamma_{n e}$ is the decay rate. Figure 3 shows the two basic decay forms of $\tilde{U}_{n}(t)$ for $n=10$ : one is the exponential decay (2.8) and the other is the algebraic decay

$$
\tilde{U}_{n}(t) \simeq \frac{1}{1+\left(\gamma_{n a} t\right)^{2}} \quad \text { for } \quad t<1 / \gamma_{n e}
$$

in the initial regime, where the numerical values of the decay rates $\gamma_{n e}, \gamma_{n a}$ and the amplitude $\alpha_{n e}$ have been determined so that Eqs. (2.8) and (2.9) fit the DNS of $\tilde{U}_{n}(t)$, and they have been listed in Table I with $1 / \gamma_{n e} \approx 9.26$ for $n=10$.

Figure 3 indicates that the DNS of $\tilde{U}_{n}(t)$ consists of the algebraic decay (2.9) in the initial regime and the exponential decay (2.8) in the final regime. Such dual structures seem to hold for $n \lesssim 15$. As will be shown later, this exponential decay (2.8) is brought about by the energy dissipation due to the chaos-induced friction and viscosity. In strong contrast to this, the algebraic decay (2.9) in the initial regime is produced by the dynamical coherence that is reversible in time.

Thus it turns out that the decay of the time correlation function $\tilde{U}_{n}(t)$ consists of the two basic forms: the exponential decay (2.8) and the algebraic decay (2.9), as shown in Fig. 3 with $\gamma_{n a} \approx \gamma_{n e} \approx 0.108$ for $n=10$. Such an approximation of $\tilde{U}_{n}(t)$ by the two basic forms (2.8) and (2.9) seems to become more important as the wave number becomes smaller.

The power spectrum $I_{\hat{u}_{n}}(\omega)$ of the time series $\hat{u}_{n}(t)$ is related to the time correlation function $\tilde{U}_{n}(t)$ by the WienerKhintchine theorem [5] as follows:

TABLE I. Numerical values of the parameters in Eqs. (2.8), (2.9), (5.3), and (4.3).

\begin{tabular}{lccccc}
\hline \hline$n$ & $\alpha_{n e}$ & $\gamma_{n e}$ & $\bar{\gamma}_{n e}$ & $\gamma_{n a}$ & $\gamma_{n g}$ \\
\hline 55 & 3.122 & 0.223 & 0.204 & 0.130 & 0.116 \\
10 & 1.755 & 0.108 & 0.123 & 0.083 & 0.077 \\
5 & 1.164 & 0.029 & & 0.041 & 0.041 \\
\hline \hline
\end{tabular}




$$
\begin{aligned}
I_{\hat{u}_{n}}(\omega) & \equiv \lim _{\tau \rightarrow \infty} \frac{\tau}{2 \pi}\left\langle\left|\frac{1}{\tau} \int_{0}^{\tau} \hat{u}_{n}(t) e^{-i \omega t} d t\right|^{2}\right\rangle \\
& =\frac{1}{\pi}\left\langle\left|\hat{u}_{n}\right|^{2}\right\rangle \tilde{U}_{n}^{\prime}(\omega),
\end{aligned}
$$

where $\widetilde{U}_{n}^{\prime}(\omega)$ is the real part of the Fourier-Laplace transform of $\tilde{U}_{n}(t)$,

$$
\tilde{U}_{n}^{\prime}(\omega) \equiv \int_{0}^{\infty} \tilde{U}_{n}(t) \cos (\omega t) d t
$$

Therefore, it becomes possible to clarify the dynamic structures of the power spectrum $I_{\hat{u}_{n}}(\omega)$ in the $(n, \omega)$ space by exploring the time correlation functions $\tilde{U}_{n}(t)$, as will be carried out in Sec. V explicitly. Equation (2.11) is one of the fluctuation-dissipation theorems.

\section{MEMORY SPECTRUM $\Gamma_{n}(i \omega)=\Gamma_{n}^{\prime}(\omega)+i \Gamma_{n}^{\prime \prime}(\omega)$}

Let us assume that the spatial period $L$ is sufficiently large so that we have the statistical homogeneity. Then, as shown in Appendix A, the time evolution of $\tilde{U}_{n}(t)$ is governed by the non-Markovian linear evolution equation [1]

$$
\frac{d \tilde{U}_{n}(t)}{d t}=-\int_{0}^{t} \Gamma_{n}(s) \tilde{U}_{n}(t-s) d s
$$

with a memory function $\Gamma_{n}(t)$ that represents the mixing and the energy dissipation due to chaos and turbulence. This is integrated to give the time correlation spectrum

$$
\tilde{U}_{n}(i \omega) \equiv \int_{0}^{\infty} \tilde{U}_{n}(t) e^{-i \omega t} d t=\frac{1}{i \omega+\Gamma_{n}(i \omega)},
$$

where we have defined the memory spectrum

$$
\Gamma_{n}(i \omega) \equiv \int_{0}^{\infty} \Gamma_{n}(t) e^{-i \omega t} d t=\Gamma_{n}^{\prime}(\omega)+i \Gamma_{n}^{\prime \prime}(\omega) .
$$

Equation (3.2) indicates that the real part $\Gamma_{n}^{\prime}(\omega)$ of the memory spectrum (3.3) represents the decay rate of $\tilde{U}_{n}(t)$ due to the chaos-induced friction and viscosity, so that $\Gamma_{n}^{\prime}(\omega)$ must be positive. Indeed, the real part of Eq. (3.2), i.e.,

$$
\tilde{U}_{n}^{\prime}(\omega)=\frac{\Gamma_{n}^{\prime}(\omega)}{\left[\omega+\Gamma_{n}^{\prime \prime}(\omega)\right]^{2}+\left[\Gamma_{n}^{\prime}(\omega)\right]^{2}}
$$

expresses the time correlation spectrum $\tilde{U}_{n}^{\prime}(\omega)$ in terms of the memory spectra $\Gamma_{n}^{\prime}(\omega)$ and $\Gamma_{n}^{\prime \prime}(\omega)$, and describes the dynamic structure of the time correlation function (2.7), leading to a generalization of the dynamic structure factor $S(\omega, k)$ of the thermal hydrodynamic fluctuations $[9,10]$. In the following, therefore, we attempt to clarify the structures of $\tilde{U}_{n}^{\prime}(\omega)$ and $\tilde{U}_{n}^{\prime \prime}(\omega)$ in terms of $\Gamma_{n}^{\prime}(\omega)$ and $\Gamma_{n}^{\prime \prime}(\omega)$ by using Eqs. (3.2) and (3.4).

The DNS of the memory spectra $\Gamma_{n}^{\prime}(\omega)$ and $\Gamma_{n}^{\prime \prime}(\omega)$ has been obtained from $\tilde{U}_{n}(t)$ by using Eq. (3.2), and is shown in
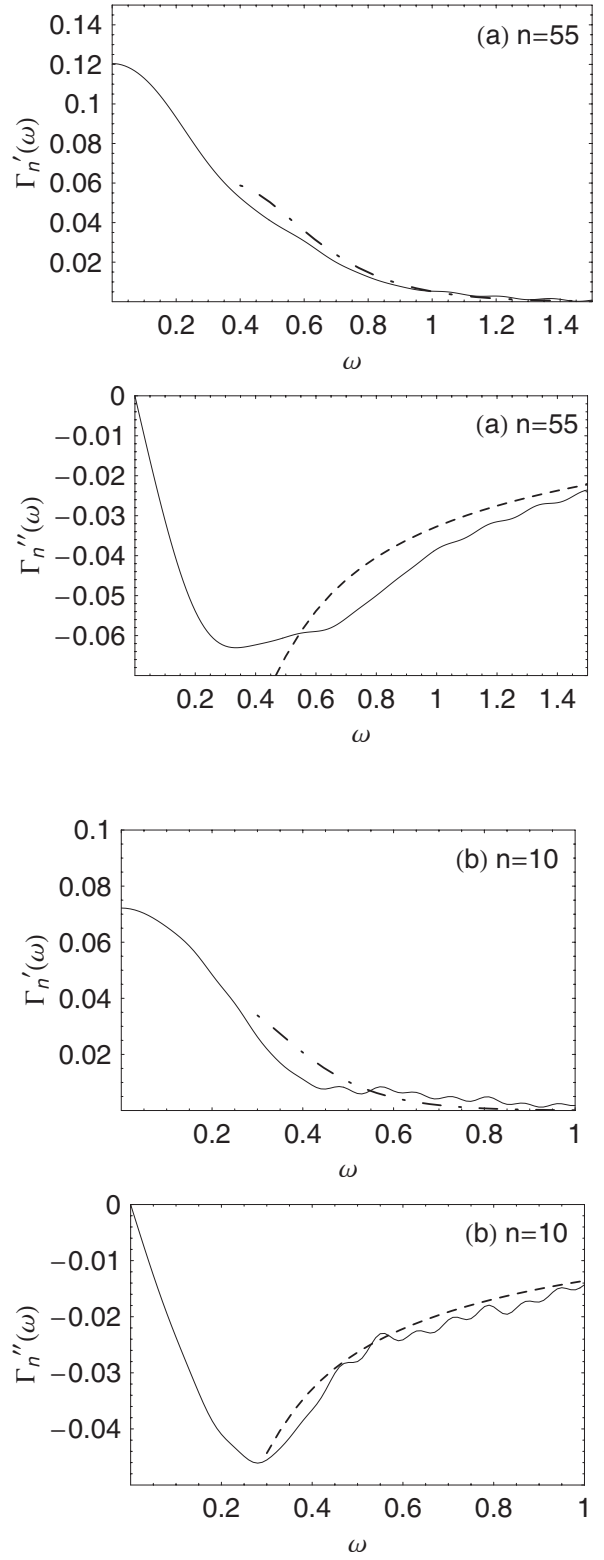

FIG. 4. DNS of the memory spectra $\Gamma_{n}^{\prime}(\omega)$ and $\Gamma_{n}^{\prime \prime}(\omega)$ for (a) $n=55$ and (b) $n=10$. The dotted-and-dashed and the broken lines denote the asymptotic forms (5.9) and (5.10), respectively.

Fig. 4 where the small-amplitude oscillations in $\omega>0.4$ seem to vanish if one takes the "ensemble" number $M$ of Eq. (2.6) sufficiently large. This indicates that $\Gamma_{n}^{\prime}(\omega)$ has a maximum at $\omega=0$, and $\Gamma_{n}^{\prime \prime}(\omega=0)=0$. In the neighborhood of $\omega$ $=0, \Gamma_{n}^{\prime}(\omega)$ and $\Gamma_{n}^{\prime \prime}(\omega)$ have the following properties:

$$
\begin{gathered}
\omega_{0}=-\Gamma_{n}^{\prime \prime}\left(\omega_{0}\right)=0,\left.\quad \Gamma_{n 1}^{\prime \prime} \equiv \frac{d \Gamma_{n}^{\prime \prime}}{d \omega}\right|_{\omega=0}<0, \\
\Gamma_{n 0}^{\prime} \equiv \Gamma_{n}^{\prime}(\omega=0)>0,\left.\quad \Gamma_{n 1}^{\prime} \equiv \frac{d \Gamma_{n}^{\prime}}{d \omega}\right|_{\omega=0}=0, \\
\left.\Gamma_{n 2}^{\prime} \equiv \frac{d^{2} \Gamma_{n}^{\prime}}{d \omega^{2}}\right|_{\omega=0}<0,
\end{gathered}
$$

where we have defined the frequency $\omega_{0}$ by $\omega_{0}+\Gamma_{n}^{\prime \prime}\left(\omega_{0}\right)=0$ in 
TABLE II. Numerical values of the decay parameters in Eqs. (3.5)-(3.8).

\begin{tabular}{lccccc}
\hline \hline$n$ & $\Gamma_{n 0}^{\prime}$ & $\Gamma_{n 1}^{\prime}$ & $\Gamma_{n 2}^{\prime}$ & $\Gamma_{n 0}^{\prime \prime}$ & $\Gamma_{n 1}^{\prime \prime}$ \\
\hline 55 & 0.119 & 0 & -1.287 & 0 & -0.298 \\
10 & 0.073 & 0 & -2.198 & 0 & -0.283 \\
\hline \hline
\end{tabular}

order to explore Eq. (3.4) theoretically. Therefore, expanding the memory spectra (3.3) into the Taylor series around the maximum $\omega=0$, we obtain

$$
\begin{gathered}
\Gamma_{n}^{\prime}(\omega)=\Gamma_{n 0}^{\prime}+\frac{1}{2} \Gamma_{n 2}^{\prime} \omega^{2}+O\left(\omega^{3}\right), \\
\Gamma_{n}^{\prime \prime}(\omega)=\Gamma_{n 1}^{\prime \prime} \omega+O\left(\omega^{2}\right) .
\end{gathered}
$$

The numerical values of the decay parameters involved in Eqs. (3.5)-(3.8) are listed in Table II for $n=55$, 10, where we have $\Gamma_{n}^{\prime}(0)=\Gamma_{n 0}^{\prime} \approx \gamma_{n a}$. The asymptotic forms of the spectra $\Gamma_{n}^{\prime}(\omega)$ and $\Gamma_{n}^{\prime \prime}(\omega)$ for $\omega \rightarrow \infty$ will be discussed in Sec. V.

\section{DERIVATION OF THE LORENTZIAN SPECTRUM}

Inserting Eqs. (3.7) and (3.8) into the basic formula (3.4), and neglecting $O\left(\omega^{2}\right)$ in the numerator and $O\left(\omega^{3}\right)$ in the denominator, we obtain

$$
\tilde{U}_{n}^{\prime}(\omega) \simeq \frac{\Gamma_{n 0}^{\prime}}{\left(1+\Gamma_{n 1}^{\prime \prime}\right)^{2} \omega^{2}+\left(\Gamma_{n 0}^{\prime}\right)^{2}+\Gamma_{n 0}^{\prime} \Gamma_{n 2}^{\prime} \omega^{2}}
$$

for $\omega \lesssim \Gamma_{n 0}^{\prime}$. This can be written as

$$
\tilde{U}_{n}^{\prime}(\omega) \simeq \frac{1}{\Gamma_{n 0}^{\prime}} \frac{\bar{\gamma}_{n e}^{2}}{\omega^{2}+\bar{\gamma}_{n e}^{2}}=\tilde{U}_{n}^{\prime}(0) \frac{\bar{\gamma}_{n e}^{2}}{\omega^{2}+\bar{\gamma}_{n e}^{2}},
$$

where we have defined the decay rate

$$
\bar{\gamma}_{n e} \equiv \frac{\Gamma_{n 0}^{\prime}}{\left[\left(1+\Gamma_{n 1}^{\prime \prime}\right)^{2}+\Gamma_{n 0}^{\prime} \Gamma_{n 2}^{\prime}\right]^{1 / 2}} .
$$

The numerical values of $\bar{\gamma}_{n e}$ are listed in Table I, which shows the reasonable relation $\bar{\gamma}_{n e} \approx \gamma_{n e}$. Since $\Gamma_{n 0}^{\prime} \approx \gamma_{n a}$ as Table II indicates, Eq. (4.3) leads to

$$
\frac{\gamma_{n a}}{\gamma_{n e}} \approx\left[\left(1+\Gamma_{n 1}^{\prime \prime}\right)^{2}+\Gamma_{n 0}^{\prime} \Gamma_{n 2}^{\prime}\right]^{1 / 2}
$$

The power spectrum of the time series $\hat{u}_{n}(t)$ is given by Eq. (2.11). Therefore, Eq. (4.2) leads to the well-known Lorentzian spectrum with half linewidth $\bar{\gamma}_{n e}$, which is equivalent to the exponential decay (2.8) with $\gamma_{n e} \approx \bar{\gamma}_{n e}$. It should be also noted that the spectrum $\tilde{U}_{n}^{\prime}(\omega)$ around $\omega=0$ becomes Lorentzian if and only if $\Gamma_{n}^{\prime}(\omega)$ has an extremum at $\omega=0$.

\section{DYNAMIC STRUCTURES OF $\tilde{U}_{n}^{\prime}(\omega)$ AND $\Gamma_{n}^{\prime}(\omega)$}

By taking the Fourier-Laplace transform of Eqs. (2.8) and (2.9), we find that the spectrum (3.4) takes the form

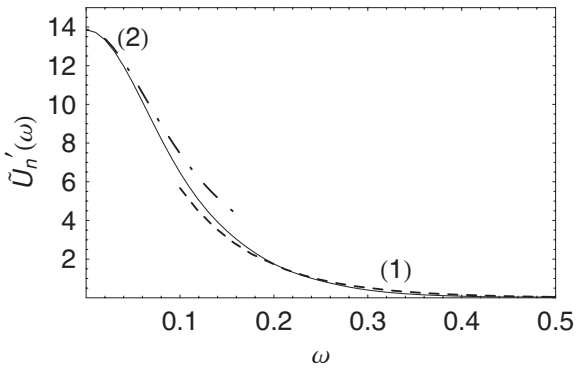

FIG. 5. The peak (2) and wing (1) of $\tilde{U}_{n}^{\prime}(\omega)$ for $n=10$ : (1) - - -, the exponential spectrum $(5.2) ;(2)-\cdot-$, the Lorentzian spectrum $(5.1) ;-$, DNS, where $\gamma_{n e} \approx 0.108$.

$\tilde{U}_{n}^{\prime}(\omega) \simeq\left\{\begin{array}{cl}\tilde{U}_{n}^{\prime}(0) \frac{1}{1+\left(\omega / \gamma_{n e}\right)^{2}} & \text { for } \omega \lesssim \gamma_{n e}, \\ \frac{\pi}{2 \gamma_{n a}} \exp \left(-\frac{\omega}{\gamma_{n a}}\right) & \text { for } \omega>\gamma_{n e},\end{array}\right.$

where Eq. (5.1) is equivalent to Eq. (4.2) with $\gamma_{n e} \approx \bar{\gamma}_{n e}$. The power spectrum of the time series $\hat{u}_{n}(t)$ is given by Eq. (2.11), and, as shown in Fig. 5, $\tilde{U}_{n}^{\prime}(\omega)$ consists of two types of structures: the Lorentzian spectrum (5.1) formed by the peak (2) of Fig. 5 and the exponential spectrum (5.2) produced by the wing (1) of Fig. 5.

Figure 6 shows $\ln \tilde{U}_{n}^{\prime}(\omega)$ against $\omega$ for (a) $n=55$ and (b) $n=10$, indicating that $\tilde{U}_{n}^{\prime}(\omega)$ with $\omega>\gamma_{n e}$ can be approximated by the exponential spectrum (5.2) much better than the Gaussian spectrum
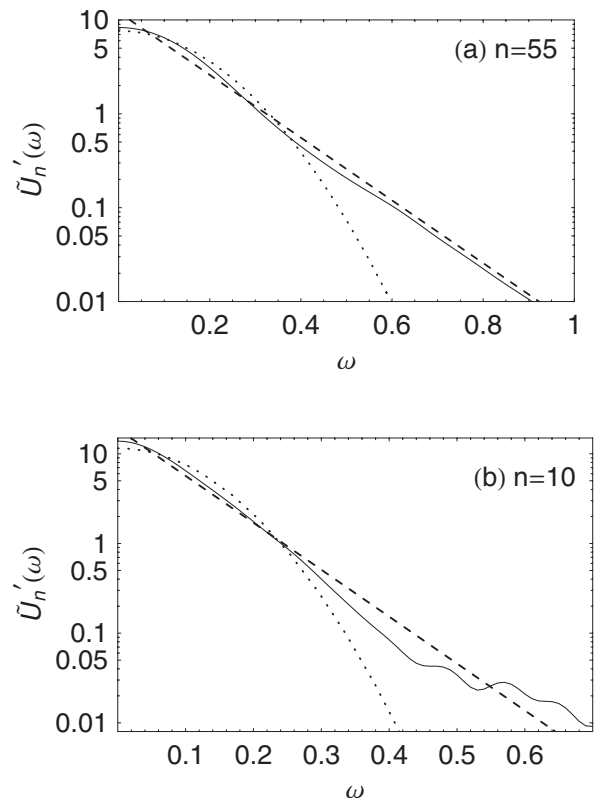

FIG. 6. $\tilde{U}_{n}^{\prime}(\omega)$ vs $\omega$ for (a) $n=55$ and (b) $n=10$ : - - , the exponential spectrum $(5.2) ; \cdots$, the Gaussian spectrum (5.3); DNS. 


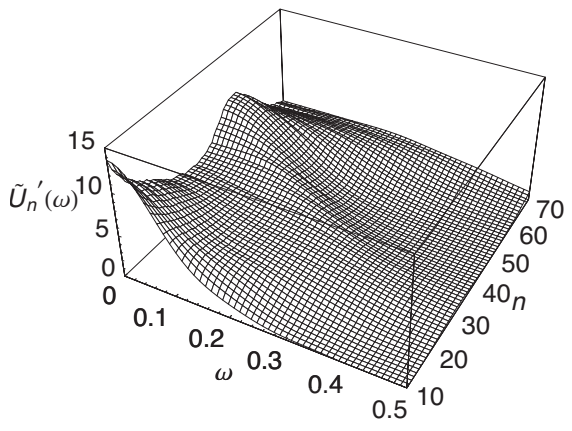

FIG. 7. Dynamic structures of $\tilde{U}_{n}^{\prime}(\omega)$ on the $\omega-n$ plane.

$$
\tilde{U}_{n g}^{\prime}(\omega) \equiv \frac{\sqrt{\pi}}{2 \gamma_{n g}} \exp \left[-\left(\frac{\omega}{2 \gamma_{n g}}\right)^{2}\right],
$$

where the numerical values of the decay rate $\gamma_{n g}$, listed in Table I, have been determined so that $\widetilde{U}_{n g}(t)=\exp \left[-\left(\gamma_{n g} t\right)^{2}\right]$ fits the DNS of $\tilde{U}_{n}(t)$ in the neighborhood of $t=0$.

Figure 5 is just the complement of Fig. 3, and their dynamic structures on the $\omega-n$ plane are shown in Fig. 7. Their most outstanding feature is the fact that $\widetilde{U}_{n}^{\prime}(\omega)$ has a dominant maximum at $\omega=0$ and is described by the Lorentzian spectrum (5.1) for $\omega \lesssim \gamma_{n e}$, as shown in Fig. 5. According to Eq. (5.2), the basic formula (3.4) must have the exponential spectrum $\exp \left(-\omega / \gamma_{n a}\right)$ for $\omega>\gamma_{n e}$, leading to the wing of Fig. 5.

The exponential spectrum (5.2) appears when the dynamical coherence is dominant, whereas the Lorentzian spectrum (5.1) occurs in a small frequency region $\omega \lesssim \gamma_{n e}$ where the dissipation rate $\gamma_{n e} \approx \bar{\gamma}_{n e}$ of Eq. (4.3) due to the chaosinduced friction and viscosity becomes dominant. Therefore, it can be concluded that the decay form of $\widetilde{U}_{n}(t)$ exhibits the dual structures, the exponential decay (2.8) and the algebraic decay (2.9), in order to bring about the two different spectra, the Lorentzian peak (5.1) and the exponential wing (5.2), respectively. Since Eqs. (5.1) and (5.2) are given by the Fourier-Laplace transform of Eqs. (2.8) and (2.9), $\tilde{U}_{n}^{\prime}(\omega)$ takes a form similar to the dual structures of $\tilde{U}_{n}(t)$. This represents the symmetry of the dual structures of chaos.

Figure 8 shows the dynamic structures of $\tilde{U}_{n}^{\prime}(\omega)$ and $\tilde{U}_{n}^{\prime \prime}(\omega)$ that are the real and imaginary parts of $\tilde{U}_{n}(i \omega)$ $=\tilde{U}_{n}^{\prime}(\omega)+i \tilde{U}_{n}^{\prime \prime}(\omega)$. Other $n$ 's give similar types of structures. The imaginary part $\tilde{U}_{n}^{\prime \prime}(\omega)$ of Eq. (3.2) takes the form

$$
\tilde{U}_{n}^{\prime \prime}(\omega)=-\frac{1}{\omega}-\frac{2 \gamma_{n a}^{2}}{\omega^{3}}+O\left(\frac{1}{\omega^{5}}\right) \text { for } \omega \rightarrow \infty,
$$

as proved in Appendix B. The asymptotic forms (5.2) and (5.4) are shown by the dotted-and-dashed and broken lines, respectively, in Fig. 8.

Next, let us consider the time evolution of the memory function $\Gamma_{n}(t)$ which is given by the time correlation function of the fluctuating force $r_{n}(t)$. The time evolution of $r_{n}(t)$ is governed by the modified propagator $\exp (t \mathcal{Q} \Lambda)$ with the projection operator $\mathcal{Q}$ that excludes the linear motion in $\hat{u}_{n}(t)$, so
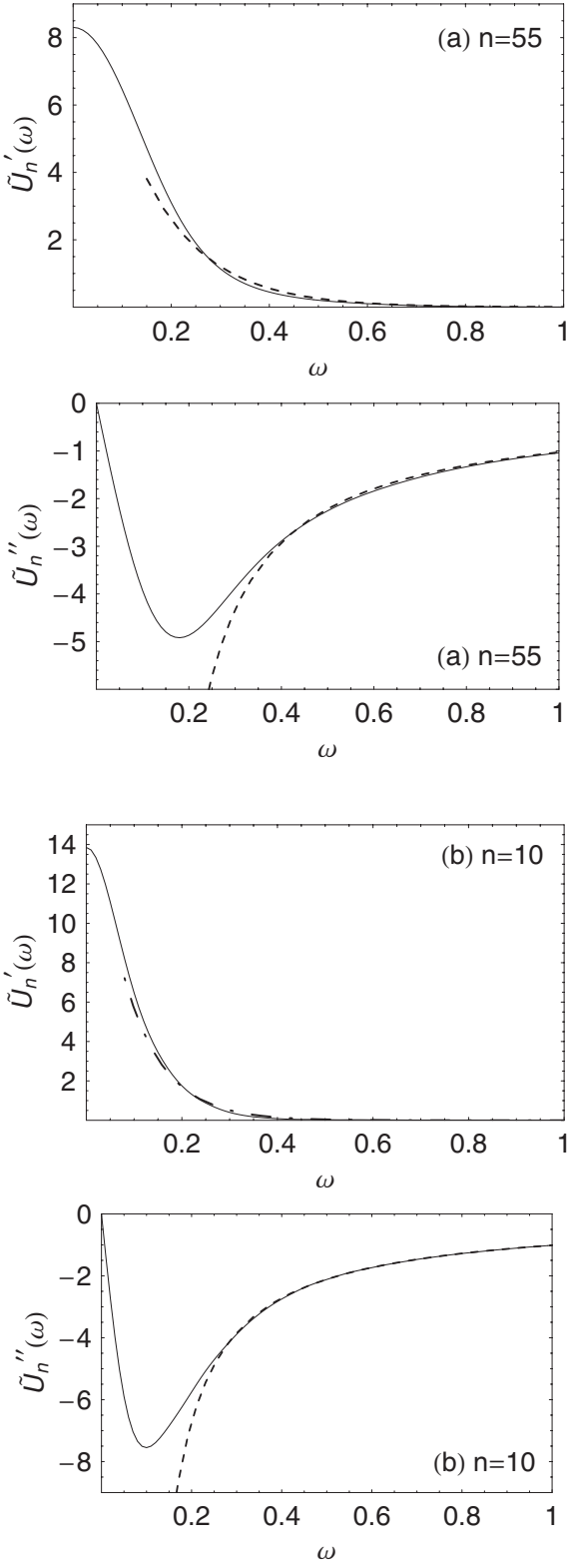

FIG. 8. Dynamic structures of $\tilde{U}_{n}^{\prime}(\omega)$ and $\tilde{U}_{n}^{\prime \prime}(\omega)$ for (a) $n=55$ and (b) $n=10$. The dotted-and-dashed and the broken lines denote the asymptotic forms (5.2) and (5.4), respectively.

that the time evolution of $\Gamma_{n}(t)$ must be different from that of $\widetilde{U}_{n}(t)$, as discussed in the previous paper [2].

Indeed, the normalized memory function $\widetilde{\Gamma}_{n}(t)$ $\equiv \Gamma_{n}(t) / \Gamma_{n}(0)$ does not have the exponential decay $\exp \left(-\gamma_{n e} t\right)$, as shown in Figs. 9 and 10, because we have excluded the linear motion in $\hat{u}_{n}$ from the time evolution of $\widetilde{\Gamma}_{n}(t)$. This is in strong contrast to the time evolution of $\tilde{U}_{n}(t)$ that always has the exponential tail. Namely, $\widetilde{\Gamma}_{n}(t)$ with large $n$ such as $n=55,30$ is given by the Gaussian decay

$$
\widetilde{\Gamma}_{n}(t) \simeq \exp \left[-\left(\beta_{n g} t\right)^{2}\right],
$$

whereas $\widetilde{\Gamma}_{n}(t)$ with small $n$ such as $n=10,5$ is given by the $3 / 2$ power decay 

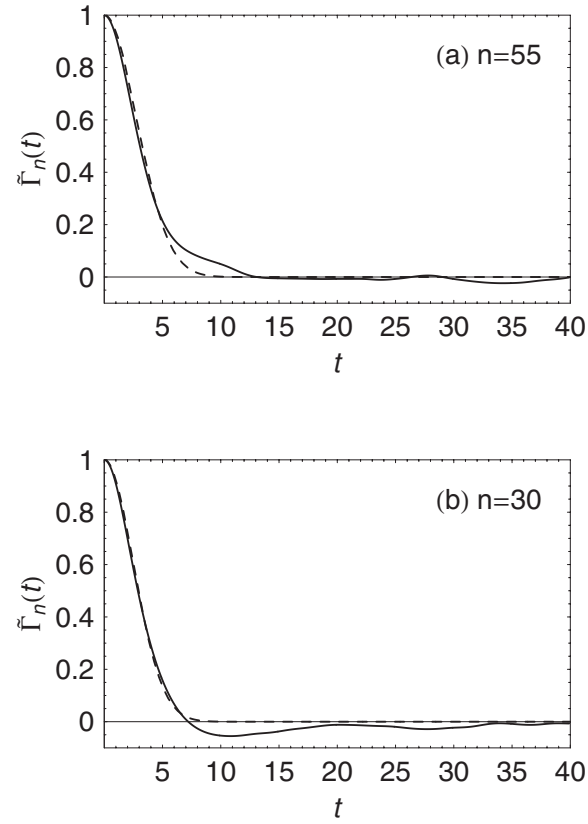

FIG. 9. Decay form of $\widetilde{\Gamma}_{n}(t)$ for (a) $n=55$ and (b) $n=30$ : - - -, the Gaussian decay (5.5); - DNS.

$$
\widetilde{\Gamma}_{n}(t) \simeq \exp \left[-\left(\beta_{n 3} t\right)^{3 / 2}\right]
$$

where the numerical values of $\beta_{n g}$ and $\beta_{n 3}$ are listed in Table III, and lead to the decay times $\tau_{n r}$ of $\widetilde{\Gamma}_{n}(t)$ lying between $1 / 0.288 \approx 3.5$ and $1 / 0.147 \approx 6.8$. In either case, the decay of $\widetilde{\Gamma}_{n}(t)$ is faster than the exponential decay $\exp \left(-\gamma_{n e} t\right)$. By comparing Fig. 10 with Fig. 1, we find in the case of $n=5$ that the decay time $\tau_{n r}\left(=1 / \beta_{n 3}\right)$ of $\widetilde{\Gamma}_{n}(t)$ is much smaller than the decay time $\tau_{n M}\left(=1 / \gamma_{n e}\right)$ of $\widetilde{U}_{n}(t)$, where Tables I and III
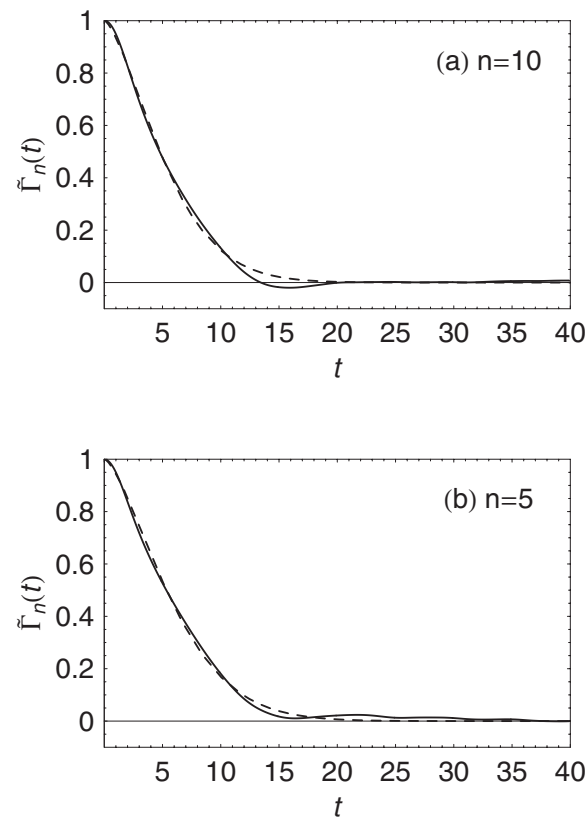

FIG. 10. Decay form of $\widetilde{\Gamma}_{n}(t)$ for (a) $n=10$ and (b) $n=5$ : - - -, the $3 / 2$ power decay $(5.6)$; - , DNS.
TABLE III. Numerical values of $\beta_{n g}$ and $\beta_{n 3}$.

\begin{tabular}{lccccc}
\hline \hline$n$ & 70 & 55 & 30 & 10 & 5 \\
\hline$\beta_{n g}$ & 0.288 & 0.255 & 0.283 & & \\
$\beta_{n 3}$ & & & & 0.164 & 0.147 \\
\hline \hline
\end{tabular}

give $\tau_{n r}=6.80$ and $\tau_{n M}=34.5$ with $\tau_{n r} / \tau_{n M}=0.20$. Therefore, if the wave number $k_{n}=2 \pi n / L$ is small enough, then we may put $\Gamma_{n}(s)=2 \Gamma_{n M} \delta(s)$ in Eq. (3.1), so that we have a Markov process $\tilde{U}_{n}(t)=\exp \left(-\Gamma_{n M} t\right)$. This is related to the eddy viscosity $\nu_{T}$ by $\Gamma_{n M}=\bar{\gamma}_{n e}=\nu_{T} k_{n}^{2}$ for $k_{n} \rightarrow 0[1]$.

Equation (3.2) leads to

$$
\begin{gathered}
\Gamma_{n}^{\prime}(\omega)=\frac{\tilde{U}_{n}^{\prime}(\omega)}{\left[\tilde{U}_{n}^{\prime}(\omega)\right]^{2}+\left[\tilde{U}_{n}^{\prime \prime}(a)\right]^{2}}, \\
\Gamma_{n}^{\prime \prime}(\omega)=-\omega-\frac{\tilde{U}_{n}^{\prime \prime}(\omega)}{\left[\tilde{U}_{n}^{\prime}(\omega)\right]^{2}+\left[\tilde{U}_{n}^{\prime \prime}(a)\right]^{2}} .
\end{gathered}
$$

Therefore, using the asymptotic forms (5.2) and (5.4) of $\tilde{U}_{n}^{\prime}(\omega)$ and $\tilde{U}_{n}^{\prime \prime}(\omega)$ for $\omega \rightarrow \infty$, we can estimate the asymptotic forms of $\Gamma_{n}^{\prime}(\omega)$ and $\Gamma_{n}^{\prime \prime}(\omega)$ for $\omega \rightarrow \infty$. Thus, inserting Eqs. (5.2) and (5.4) into Eq. (5.7), we obtain

$$
\Gamma_{n}^{\prime}(\omega) \rightarrow \frac{\tilde{U}_{n}^{\prime}(\omega)}{\left[\tilde{U}_{n}^{\prime \prime}(\omega)\right]^{2}} \rightarrow \frac{\pi}{2 \gamma_{n a}} \omega^{2} \exp \left(-\frac{\omega}{\gamma_{n a}}\right)
$$

for $\omega \rightarrow \infty$. Similarly, inserting Eqs. (5.2) and (5.4) into Eq. (5.8), we obtain

$$
\Gamma_{n}^{\prime \prime}(\omega) \rightarrow-\omega-\frac{1}{\tilde{U}_{n}^{\prime \prime}(\omega)} \rightarrow-\frac{2 \gamma_{n a}^{2}}{\omega}\left(1-\frac{2 \gamma_{n a}^{2}}{\omega^{2}}\right)
$$

for $\omega \rightarrow \infty$. The asymptotic forms (5.9) and (5.10) are shown by the dotted-and-dashed and broken lines, respectively, in Fig. 4. These asymptotic forms of $\tilde{U}_{n}(i \omega)$ and $\Gamma_{n}(i \omega)$ are useful for clarifying the dynamic structures for $\omega \rightarrow \infty$.

\section{STOCHASTIC FREQUENCY MODULATION MODEL}

Finally, let us discuss a stochastic model that brings about both the exponential decay (2.8) and the algebraic decay (2.9) theoretically. That is the frequency modulation model $d z(t) / d t=i \Omega(t) z(t)$ [5], where $\Omega(t)$ is a Gaussian stochastic process with $\langle\Omega(t)\rangle=0$. The initial value $z_{0}=z(t=0)$ is assumed to be independent of the process $\Omega(t)$. This model has been invented as a simplified model for discussing the magnetic resonance absorption, in particular, the nuclear magnetic resonance absorption $[5,12]$. Then we have [5]

$$
\tilde{U}(t) \equiv \frac{\left\langle z(t) z^{*}(0)\right\rangle}{\left\langle\left|z_{0}\right|^{2}\right\rangle}=\exp \left[-\int_{0}^{t}(t-s)\langle\Omega(s) \Omega(0)\rangle d s\right] \text {. }
$$

We now assume that [12] 


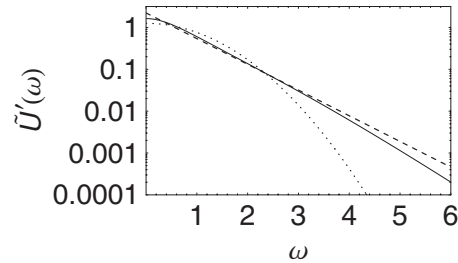

FIG. 11. Time correlation spectra of the stochastic model (6.1): —, the exact spectrum $\tilde{U}^{\prime}(\omega)$ obtained from Eq. (6.3); - - -, the exponential spectrum $\widetilde{U}_{a}^{\prime}(\omega) ; \cdots$, the Gaussian spectrum $\widetilde{U}_{g}^{\prime}(\omega)$.

$$
\langle\Omega(t) \Omega(0)\rangle=e^{-t^{2}} .
$$

Then, Eq. (6.1) can be expressed by

$$
\tilde{U}(t)=\exp \left[-t \int_{0}^{t} e^{-x^{2}} d x+\frac{1}{2}\left(1-e^{-t^{2}}\right)\right] .
$$

This reduces to $\tilde{U}(t) \simeq \exp (-\sqrt{\pi} t / 2)$ for $t \gg 1$.

Figure 11 shows three time correlation spectra: the exact spectrum $\tilde{U}^{\prime}(\omega)$, the exponential spectrum

$$
\tilde{U}_{a}^{\prime}(\omega)=\frac{\pi}{\sqrt{2}} \exp (-\sqrt{2} \omega)
$$

and the Gaussian spectrum

$$
\tilde{U}_{g}^{\prime}(\omega)=\sqrt{\frac{\pi}{2}} \exp \left(-\omega^{2} / 2\right)
$$

which are the real parts of the Fourier-Laplace transforms of the time correlation functions: $\widetilde{U}(t)$ in Eq. (6.3), the algebraic decay

$$
\tilde{U}_{a}(t)=\frac{1}{1+(t / \sqrt{2})^{2}},
$$

and the Gaussian decay

$$
\tilde{U}_{g}(t)=\exp \left[-(t / \sqrt{2})^{2}\right],
$$

respectively. The figure indicates that the exponential spectrum $\tilde{U}_{a}^{\prime}(\omega)$ agrees with the exact spectrum obtained from Eq. (6.3) much better than the Gaussian spectrum $\tilde{U}_{g}^{\prime}(\omega)$ for larger $\omega$. This means that the time correlation function of the form (6.3) can be approximated by the algebraic decay $\tilde{U}_{a}(t)$ much better than the Gaussian decay $\tilde{U}_{g}(t)$ for smaller $t$. Therefore we have

$$
\tilde{U}(t) \simeq \begin{cases}\frac{1}{1+(t / \sqrt{2})^{2}} & \text { for } t \leqq \sqrt{2}, \\ \exp (-\sqrt{\pi} t / 2) & \text { for } t \gg 1 .\end{cases}
$$

Equations (6.8) and (6.9) lead to the exponential decay (2.8) and the algebraic decay (2.9), by taking the algebraic decay $\widetilde{U}_{a}(t)$ in contrast to the conventional theory [5] that takes the Gaussian decay $\tilde{U}_{g}(t)$. The stochastic frequency modulation model would, therefore, suggest that the dual structures of the exponential decay (2.8) and the algebraic decay (2.9) hold quite generally beyond the chaotic KS equation.

\section{SUMMARY}

It has been shown for the KS equation (2.1) that the time correlation function (2.7) exhibits the two decay forms, the exponential decay (2.8), and the algebraic decay (2.9), leading to the two basic structures of Fig. 3. These dynamic structures may be summarized by Figs. 5 and 7 for the time correlation spectrum $\tilde{U}_{n}^{\prime}(\omega)$, and have been clarified by integrating the non-Markovian linear evolution Eq. (3.1). Thus we have discovered that the decay form of the time correlation function (2.7) exhibits the dual structures, the exponential decay (2.8) and the algebraic decay (2.9), which bring about the universal Lorentzian peak (5.1) and the exponential wing (5.2), respectively. Namely, $\tilde{U}_{n}^{\prime}(\omega)$ takes a form similar to $\widetilde{U}_{n}(t)$, which represents the symmetry of the dual structures of chaos. Thus we have found the following four basic decay forms.

(1) The time correlation function $\tilde{U}_{n}(t)$ consists of the algebraic decay $1 /\left[1+\left(\gamma_{n a} t\right)^{2}\right]$ for $t<1 / \gamma_{n e}$ and the exponential decay $\exp \left(-\gamma_{n e} t\right)$ for $t>1 / \gamma_{n e}$, as shown in Fig. 3. In contrast to this, the memory function $\Gamma_{n}(t)$ takes the Gaussian decay $\exp \left[-\left(\beta_{n g} t\right)^{2}\right]$ for overall time in the case of large wave numbers, but the $3 / 2$ power decay $\exp \left[-\left(\beta_{n 3} t\right)^{3 / 2}\right]$ in the case of small wave numbers. Thus it has turned out that the basic decay forms consist of the algebraic decay $1 /[1$ $\left.+\left(\gamma_{n a} t\right)^{2}\right]$ and the following three types: $\exp \left[-\left(\beta_{n m} t\right)^{m / 2}\right](m$ $=2,3,4)$.

This conclusion differs from the conventional theory of the random frequency modulation [5] that takes the Gaussian decay $\exp \left[-\left(\gamma_{n g} t\right)^{2}\right]$ for $t<1 / \gamma_{n e}$ instead of the algebraic decay $1 /\left[1+\left(\gamma_{n a} t\right)^{2}\right]$. As shown in Figs. 11 and 6, however, we have to take the algebraic decay or equivalently the exponential spectrum (5.2), since this gives a better approximation than the Gaussian decay or equivalently the Gaussian spectrum (5.3).

(2) If the wave number is small enough, then the decay time $\tau_{n M}\left(=1 / \gamma_{n e}\right)$ of $\tilde{U}_{n}(t)$ becomes much larger than the decay time $\tau_{n r}\left(=1 / \beta_{n 3}\right)$ of $\Gamma_{n}(t)$, so that we may put $\Gamma_{n}(t)$ $=2 \Gamma_{n M} \delta(t)$ in Eq. (3.1). This leads to a Markov process $\tilde{U}_{n}(t)=\exp \left(-\Gamma_{n M} t\right)$. Then we have $\Gamma_{n M}=\nu_{T} k_{n}^{2}$ for $k_{n} \rightarrow 0$ with the eddy viscosity $\nu_{T}[1]$.

(3) The real part $\Gamma_{n}^{\prime}(\omega)$ of the memory spectrum (3.3) is positive and has a maximum at $\omega=0$, as shown in Fig. 4 . It is interesting that, as shown in Fig. 5, the time correlation spectrum $\tilde{U}_{n}^{\prime}(\omega)$ and the power spectrum $I_{\hat{u}_{n}}(\omega)$ have a dominant peak at $\omega=0$, at which the memory spectrum $\Gamma_{n}^{\prime}(\omega)$ has a maximum. It has been shown in Eqs. (3.4) and (4.2) that $\Gamma_{n}^{\prime}(\omega)$ represents the decay processes of $\tilde{U}_{n}(t)$ due to the chaos-induced friction and viscosity. Indeed, Eq. (4.3) would lead to $\bar{\gamma}_{n e}=\nu_{T} k_{n}^{2}$ for $k_{n} \rightarrow 0$.

(4) The imaginary parts $\tilde{U}_{n}^{\prime \prime}(\omega)$ and $\Gamma_{n}^{\prime \prime}(\omega)$ have been shown to take the forms of Figs. 8 and 4, whose asymptotic forms for $\omega \rightarrow \infty$ are given by Eqs. (5.4) and (5.10). 
There exist several studies of the time correlation functions and the power spectra for various dynamical systems, such as the Hénon-Heiles system [13,14], the OrszagMcLaughlin system [13], and the Lorenz system [15,16]. However, these papers do not explore the decay forms of the time correlation functions and the dynamic structures of the power spectra, which are the subjects of the present paper.

The real part $\tilde{U}_{n}^{\prime}(\omega)$ of Eq. (3.2) gives the power spectrum $I_{\hat{u}_{n}}(\omega)$ of the time series $\hat{u}_{n}(t)$, and represents the dynamic structures of Eq. (3.4) in terms of the memory spectra $\Gamma_{n}^{\prime}(\omega)$ and $\Gamma_{n}^{\prime \prime}(\omega)$. This equation indicates that $\widetilde{U}_{n}^{\prime}(\omega)$ gives a generalization of the dynamic structure factor $S(\omega, k)[9,10]$ of the thermal hydrodynamic fluctuations for the chaotic hydrodynamic fluctuations. Therefore, in the present paper, we have developed a modern statistical-physical approach to the dynamic structures of the chaotic hydrodynamic fluctuations, which can be measured by the scattering of light and neutrons by the hydrodynamic fluctuations.

\section{APPENDIX A: DERIVATION OF EQ. (3.1) FROM EQ. (2.2)}

In order to derive a non-Markovian linear evolution equation for $\widetilde{U}_{n}(t)$ from Eq. (2.2) exactly [1], let us employ the projection operator formalism $[2,6]$ by introducing the projection operator

$$
\mathcal{P} g(\hat{\boldsymbol{u}}(t))=\sum_{l} \sum_{m}\left\langle g(\hat{\boldsymbol{u}}(t)) \hat{u}_{l}^{*}\right\rangle\left[\left\langle\hat{\boldsymbol{u}}^{\dagger}\right\rangle^{-1}\right]_{l m} \hat{u}_{m}
$$

for $\hat{\boldsymbol{u}} \equiv\left\{\hat{u}_{n}\right\}, n=1, \ldots, N$, where $\left\langle\hat{\boldsymbol{u}} \hat{\boldsymbol{u}}^{\dagger}\right\rangle$ denotes the square matrix $\left\langle\hat{u}_{l} \hat{u}_{m}^{\dagger}\right\rangle$ and $\left\langle\hat{\boldsymbol{u}} \hat{\boldsymbol{u}}^{\dagger}\right\rangle^{-1}$ means its inverse.

Then, using the Liouville evolution operator $\Lambda$ [2] and the projection operator $\mathcal{Q}=1-\mathcal{P},(\mathcal{P Q}=0)$, we can rewrite the nonlinear force $(2.4)$ as

$$
\begin{aligned}
N_{n}(t) & =e^{t \Lambda} N_{n}(0)=e^{t \Lambda}(\mathcal{P}+\mathcal{Q}) N_{n}(0), \\
= & \sum_{m} \Omega_{n m} \hat{u}_{m}(t)+e^{t \Lambda} \mathcal{Q} N_{n}(0),
\end{aligned}
$$

where we have defined a frequency matrix

$$
\Omega_{n m} \equiv \sum_{l}\left\langle N_{n}(0) \hat{u}_{l}^{*}\right\rangle\left[\left\langle\hat{\boldsymbol{u}} \hat{\boldsymbol{u}}^{\dagger}\right\rangle^{-1}\right]_{l m} .
$$

Therefore, inserting the operator identity $[2,3]$

$$
e^{t \Lambda}=e^{t \mathcal{Q} \Lambda}+\int_{0}^{t} d s e^{s \Lambda} \mathcal{P} \Lambda e^{(t-s) \mathcal{Q} \Lambda}
$$

into the second term of Eq. (A3) and defining the fluctuating forces

$$
r_{n}(t) \equiv e^{t \mathcal{Q} \Lambda} \mathcal{Q} N_{n}(0),
$$

we obtain the new form

$$
N_{n}(t)=\sum_{m} \Omega_{n m} \hat{u}_{m}(t)-\sum_{m} \int_{0}^{t} \Gamma_{n m}(t-s) \hat{u}_{m}(s) d s+r_{n}(t),
$$

where we have defined the memory functions $[2,3]$

$$
\begin{aligned}
\Gamma_{n m}(t) & \equiv-\sum_{l}\left\langle\left\{\Lambda r_{n}(t)\right\} \hat{u}_{l}^{*}\right\rangle\left[\left\langle\hat{\boldsymbol{u}} \hat{\boldsymbol{u}}^{\dagger}\right\rangle^{-1}\right]_{l m}, \\
& =\sum_{l}\left\langle r_{n}(t) r_{l}^{*}(0)\right\rangle\left[\left\langle\hat{\boldsymbol{u}} \hat{\boldsymbol{u}}^{\dagger}\right\rangle^{-1}\right]_{l m} .
\end{aligned}
$$

Equation (A7) means that the nonlinear force $N_{n}(t)$ can be written as the sum of a linear part in $\hat{u}_{m}(s)(t \geq s>0)$ and a nonlinear fluctuating force $r_{n}(t)$ that is orthogonal to $\hat{\boldsymbol{u}}$ :

$$
\mathcal{P} r_{n}(t)=0, \quad\left\langle r_{n}(t) \hat{u}_{l}^{*}\right\rangle=0 .
$$

Thus the memory functions $\Gamma_{n m}(t)$ are given by the time correlation functions (A9) of the fluctuating forces (A6), and represent the mixing and relaxation processes of the time correlation functions $\tilde{U}_{n}(t)$ due to chaos and turbulence. Equation (A9) is often called the second fluctuationdissipation theorem $[5,6]$.

The KS equation (2.1) is invariant under the spatial translation. Then for a sufficiently large spatial period of $L$, we can assume the statistical homogeneity [1]

$$
\begin{gathered}
\left\langle\hat{u}_{n}(t) \hat{u}_{m}^{*}(s)\right\rangle=\left\langle\hat{u}_{n}(t) \hat{u}_{n}^{*}(s)\right\rangle \delta_{n m}, \\
\left\langle\hat{u}_{n}(t) \hat{u}_{m}(t) \hat{u}_{l}(s)\right\rangle=0 \quad \text { unless } \quad n+m+l=0, \\
\Omega_{n m}=\Omega_{n} \delta_{n m}, \quad\left(\Omega_{n} \equiv \Omega_{n n}\right), \\
\Gamma_{n m}(t)=\Gamma_{n}(t) \delta_{n m}, \quad\left[\Gamma_{n}(t) \equiv \Gamma_{n n}(t)\right] .
\end{gathered}
$$

As shown in Ref. [1], we further have

$$
L_{n}+\Omega_{n}=0,
$$

so that, inserting Eq. (A7) into Eq. (2.2), we obtain the evolution equation for $\hat{u}_{n}(t)$

$$
\frac{d \hat{u}_{n}(t)}{d t}=-\int_{0}^{t} \Gamma_{n}(t-s) \hat{u}_{n}(s) d s+r_{n}(t),
$$

where Eq. (A9) reduces to

$$
\Gamma_{n}(t)=\frac{\left\langle r_{n}(t) r_{n}^{*}(0)\right\rangle}{\left\langle\left|\hat{u}_{n}\right|^{2}\right\rangle} .
$$

Let us multiply Eq. (A16) by $\hat{u}_{n}^{*}(0)$ and take its average $\langle\cdots\rangle$. Then, since $\widetilde{U}_{n}(t)=\left\langle\hat{u}_{n}(t) \hat{u}_{n}^{*}(0)\right\rangle /\left\langle\left|\hat{u}_{n}\right|^{2}\right\rangle$ and $\left\langle r_{n}(t) \hat{u}_{n}^{*}(0)\right\rangle=0$, we obtain

$$
\frac{d \tilde{U}_{n}(t)}{d t}=-\int_{0}^{t} \Gamma_{n}(t-s) \tilde{U}_{n}(s) d s .
$$

This leads to Eq. (3.1).

\section{APPENDIX B: ASYMPTOTIC FORM OF $\tilde{U}_{n}(i \omega)$ FOR $\omega \rightarrow \infty$}

Taking the imaginary part of Eq. (3.2), we obtain

$$
\tilde{U}_{n}^{\prime \prime}(\omega)=-\int_{0}^{\infty} \tilde{U}_{n}(t) \sin (\omega t) d t .
$$

The partial integration of this leads to 


$$
\begin{aligned}
\widetilde{U}_{n}^{\prime \prime}(\omega)= & -\frac{1}{\omega}-\frac{1}{\omega} \int_{0}^{\infty} \frac{d \tilde{U}_{n}(t)}{d t} \cos (\omega t) d t \\
= & -\frac{1}{\omega}-\frac{1}{\omega^{3}} \int_{0}^{\infty} \frac{d}{d t}\left[\frac{d^{2} \widetilde{U}_{n}(t)}{d t^{2}} \cos (\omega t)\right] d t \\
& +\frac{1}{\omega^{3}} \int_{0}^{\infty} \frac{d^{3} \widetilde{U}_{n}(t)}{d t^{3}} \cos (\omega t) d t \\
= & -\frac{1}{\omega}+\left.\frac{1}{\omega^{3}} \frac{d^{2} \widetilde{U}_{n}(t)}{d t^{2}}\right|_{t=0}+O\left(\frac{1}{\omega^{5}}\right)
\end{aligned}
$$

for $\omega \rightarrow \infty$, where

$$
\tilde{U}_{n}(t=0)=1,\left.\quad \frac{d \tilde{U}_{n}(t)}{d t}\right|_{t=0}=0, \quad \lim _{t \rightarrow \infty} \tilde{U}_{n}(t)=0 .
$$

Since Eq. (2.9) gives

$$
\left.\frac{d^{2} \widetilde{U}_{n}(t)}{d t^{2}}\right|_{t=0}=-2 \gamma_{n a}^{2}
$$

Equation (B1) takes the form

$$
\tilde{U}_{n}^{\prime \prime}(\omega)=-\frac{1}{\omega}-\frac{2 \gamma_{n a}^{2}}{\omega^{3}}+O\left(\frac{1}{\omega^{5}}\right)
$$

for $\omega \rightarrow \infty$. This leads to Eq. (5.4). By comparing Eq. (B1) with Eq. (3.2), we find that $\Gamma_{n}(i \omega) \rightarrow 0$ as $\omega \rightarrow \infty$. Taking the real part of Eq. (3.2) and inserting Eq. (2.9), we obtain

$$
\begin{aligned}
\tilde{U}_{n}^{\prime}(\omega) & =\int_{0}^{\infty} \tilde{U}_{n}(t) \cos (\omega t) d t \simeq \int_{0}^{\infty} \frac{\cos (\omega t)}{1-\left.\frac{1}{2} \frac{d^{2} \tilde{U}_{n}(t)}{d t^{2}}\right|_{t=0} t^{2}} d t \\
& =\frac{1}{2} \int_{-\infty}^{\infty} \frac{e^{-i \omega t}}{1+\left(\gamma_{n a} t\right)^{2}} d t
\end{aligned}
$$

for $\omega \rightarrow \infty$. The integral (B4) reduces to Eq. (5.2).
[1] M. Okamura, Phys. Rev. E 74, 046210 (2006).

[2] H. Mori, S. Kuroki, H. Tominaga, R. Ishizaki, and N. Mori, Prog. Theor. Phys. 111, 635 (2004).

[3] H. Mori, S. Kuroki, and T. Horita, Prog. Theor. Phys. 113, 29 (2005).

[4] P. A. Davidson, Turbulence: An Introduction for Scientists and Engineers (Oxford University Press, Oxford, 2004).

[5] R. Kubo, M. Toda, and N. Hashitsume, Statistical Physics II, Nonequilibrium Statistical Mechanics (Springer-Verlag, Berlin, 1991).

[6] H. Mori, Prog. Theor. Phys. 33, 423 (1965).

[7] U. Balucani, M. H. Lee, and V. Tognetti, Phys. Rep. 373, 409 (2003).

[8] H. Mori and H. Fujisaka, Phys. Rev. E 63, 026302 (2001).

[9] J. P. Boon and S. Yip, Molecular Hydrodynamics (McGraw-
Hill, New York, 1980).

[10] J. M. Ortiz de Zárate and J. V. Sengers, Hydrodynamic Fluctuations in Fluids and Fluid Mixtures (Elsevier, Amsterdam, 2006).

[11] M. C. Cross and P. C. Hohenberg, Rev. Mod. Phys. 65, 851 (1993).

[12] P. W. Anderson and P. R. Weiss, Rev. Mod. Phys. 25, 269 (1953).

[13] M. Falcioni, S. Isola, and A. Vulpiani, Phys. Lett. A 144, 341 (1990).

[14] R. Ishizaki, H. Mori, H. Tominaga, S. Kuroki, and N. Mori, Prog. Theor. Phys. 116, 1051 (2006).

[15] C. H. Reick, Phys. Rev. E 66, 036103 (2002).

[16] G. Boffetta, G. Lacorata, S. Musacchio, and A. Vulpiani, Chaos 13, 806 (2003). 